\title{
Intradimensional and extradimensional shifts in the rat with assessment of differential instrumental generalization
}

\author{
CHARLES C. SPIKER and JOAN H. CANTOR \\ University of Iowa, Iowa City, Iowa 52242
}

\begin{abstract}
Intradimensional (ID) and extradimer ional (ED) shifts were compared using rats as subjects. By counterbalancing with respect to the positive cue in the shift task, it was possible to determine whether ID subjects were facilitated by favorable generalization of the instrumental response that was acquired in original learning. The results confirmed the superiority of the ID over the ED shift, previously reported by Shepp and Eimas (1964), and there was no significant evidence of differential generalization of the instrumental response. It is concluded that the Shepp and Eimas results were replicated and that the ID superiority cannot be accounted for solely in terms of favorable generalization of the instrumental response.
\end{abstract}

Several years ago, Shepp and Eimas (1964) conducted an experiment that demonstrated that rats given an intradimensional (ID) shift performed better than those given an extradimensional (ED) shift. In a follow-up experiment, Turrisi, Shepp, and Eimas (1969) reported that the advantage of the ID shift over the ED shift disappeared when the values of the irrelevant dimension in the first task were held constant for both shift groups. For comparative purposes, the authors re-presented the 1964 data rather than a new replication of them. The 1964 experiment is frequently cited to support the need for attentional theories of discrimination learning in infrahuman subjects. Despite the critical importance of the results in this connection, the Shepp and Eimas experiment apparently remains the single piece of published evidence that ID shifts are easier than ED shifts for the rat (Mackintosh, 1974).

The ID and ED paradigms, which call for a shift in dimensional values between the initial and shift tasks, were developed for investigating the transfer of dimensional responding (e.g., dimensional attention) with the transfer of the instrumental response minimized. The proponents of these paradigms (e.g., Shepp \& Turrisi, 1966) argue that changing the dimensional values between tasks minimizes the likelihood that the firsttask instrumental response will generalize to the second task. For example, an ID subject, trained with circle positive and triangle negative in Task 1 , is expected to have little or no preference for either the square or cross in Task 2. Since the ED subject's relevant dimension in Task 1 is irrelevant in Task 2, generalization from Task 1

This research was supported in part by funds supplied by the Graduate College, University of Iowa. The authors are indebted to Michael Grisham for providing laboratory space and to Edward Essock and Clarence Kreiter for assistance in collecting data. Requests for reprints should be addressed to Charles C. Spiker, Department of Psychology, University of Iowa, Iowa City, Iowa 52242. to Task 2 cannot create a preference for either relevant cue of Task 2. For the ID subject, however, there may be some generalization from the relevant first-task cues to the relevant second-task cues. To prevent mild generalization-induced preferences from systematically affecting Task 2 performance, Shepp and Eimas (1964) formed subgroups to balance the several values of the form and stripe attributes across the first and second tasks.

Other investigators (e.g., Kendler, Kendler, \& Ward, 1972) have pointed out that counterbalancing may not adequately control for differential generalization of the instrumental response for the ID subjects. The adequacy of the counterbalancing rests on an assumption that the positive and negative transfer effects are symmetrical, that is, that the effects of early reinforcement of ID subjects who are facilitated is balanced by the effects of early nonreinforcement of ID subjects who experience unfavorable generalization. Translated into theoretical considerations, symmetrical transfer effects depend upon a highly specific set of parametric assumptions. In terms of Hull-Spence theory, it would require that the increments of habit be equal to the increments in inhibition and that both increments be independent of the stage of learning. Within the attention theory that Shepp and Eimas prefer, the symmetrical transfer effects would require specific assumptions about the several parameters that govern changes in the probabilities of dimensional attention and of instrumental responses.

Irrespective of theoretical considerations, assessment of the transfer effects is a relatively straightforward matter. To determine whether differential generalization of the instrumental response has produced the ID superiority, one needs only to determine whether or not the difference between the ID and ED performances varies among the counterbalancing subgroups. If there is a significant interaction between type of shift and counterbalancing conditions, differential generalization of the instrumental response is suspect; conversely, 
absence of an interaction supports the contention that differential generalization does not account for the superiority of the ID shift over the ED shift. Unfortunately, Shepp and Eimas (1964) did not report the data separately for the counterbalancing subgroups.

The purpose of the present experiment was to attempt to replicate the critical outcome of the Shepp and Eimas (1964) experiment and to assess the degree to which differential generalization of the instrumental response may account for any ID superiority.

\section{METHOD}

\section{Subjects}

Thirty-two naive hooded male rats, 90 to 110 days of age at the start of the experiment, were obtained from the colony maintained by the Department of Psychology, University of Iowa. An additional three rats were dropped from the experiment because they refused to respond after having been inadvertently struck by the lowering door. Two of the latter subjects were originally assigned to an ID condition and the other was an ED subject.

\section{Apparatus}

The design of the apparatus employed was based on, and was functionally equivalent to, that described by Rollin, Shepp, and Thaller (1963). Six identical units were constructed side by side on a common frame. Each unit consisted of a chamber and a stimulus tray. The chamber was constructed of black Plexiglas, $15.5 \times 30 \times 20 \mathrm{~cm}$ deep, with $1 / 2$-in. hardware-cloth flooring. The top of the chamber was a hinged transparent Plexiglas door. At the end of the chamber near the stimulus tray was a removable plate of clear Plexiglas, $7.5 \mathrm{~cm}$ high and extending across the entire width of the chamber, in which two holes, $7 \mathrm{~cm}$ apart from center to center, were cut. Just outside the plate was the stimulus tray with two aluminum food wells aligned with the holes in the plate. Between the plate and the tray was an opaque door that could be manually raised and lowered between trials to provide access to the stimulus tray through the holes in the plate. A $15-\mathrm{W}$ fluorescent lamp was mounted $25 \mathrm{~cm}$ above the tray. A 16-in. electric fan was used to mask extraneous sounds.

The stimulus objects differed with respect to form and stripes. The four form stimuli (circle, square, inverted triangle, and $\mathrm{X}$ ) were cut from 3/8-in. clear Plexiglas. The diameter of the circle was $4 \mathrm{~cm}$ and the dimensions of the other stimuli were adjusted so that their areas approximated that of the circle. Stripes of different widths $(1 / 16,1 / 8$, and $1 / 4$ in.) were constructed by attaching strips of black drafting tape to white cardboard. Three layers of tape were used to provide a threedimensional effect, and epoxy glue was painted on to provide a durable surface that could be cleaned periodically. The stripes were then cut to match the various forms and were mounted in variable orientation (vertical, horizontal, or diagonal) on the front faces of the Plexiglas forms. The stimulus objects were fastened from the back to black aluminum mounts, which were in turn fastened to black Plexiglas platforms. The stimulus objects were attached to the mounts so that their top edges sloped away from the windows at an angle of about $20 \mathrm{deg}$ from the vertical. The platforms were constructed to move easily along two aluminum tracks that extended back from the chamber on either side of a food well. Thus, in the forward position, the stimulus object was directly in front of a window in the chamber plate, with the platform base covering the food well. When the rat pushed against the stimulus object, both the object and the platform were pushed back, exposing the food well.

\section{Procedure}

On the 1st day of adaptation, the rats were delivered from the colony in group cages and were individually removed and handled for 3 to $5 \mathrm{~min}$ before being placed in individual cages. Beginning the 2 nd day, they were weighed daily throughout the experiment. After the subjects were weighed on the 4 th day, all food was removed from the cages. Beginning the 5 th day, the rats were fed 8 to $14 \mathrm{~g}$ of food until they had been reduced to $85 \%$ of the average of their weights on Days $2-4$. The amount of food given subsequently was adjusted so that each rat was allowed to gain $.5 \%$ of its weight per day. Adaptation to the apparatus was begun on Day 6, when each rat was placed in a chamber for 2 to $3 \mathrm{~min}$ with a food cup containing $15(.45-\mathrm{mg})$ food pellets inside the chamber.

On the first day of pretraining, 15 food pellets were placed in the cup in the chamber and in each of the two food wells. The rat was placed in the chamber, the fluorescent lamp was turned on and the overhead lights were turned off, and the door to the stimulus tray was raised and held open for $25 \mathrm{~min}$. This procedure was repeated the following day for any subject who failed to eat all except two pellets in each of the three food cups. An occasional subject required a 3 rd day to meet this criterion.

In the second phase of pretraining, the food cups were removed from the chambers and the subjects were trained to uncover the food wells. Sixteen trials were administered each day, on each of which two food pellets were placed in each food well. A black Plexiglas upright panel, mounted on one of the platforms, was locked in place directly before one of the windows in order to direct the rat's response to the other window. A clear panel, $7 \mathrm{~cm}$ square, mounted on a platform at an angle $20 \mathrm{deg}$ from the vertical, was used to train the rats to uncover the food wells. The training panel was gradually moved forward from trial to trial until it finally fully covered the food well. On each day, half of the trials involved one food well and the other half involved the other. The criterion for this phase of training was 16 trials ( 1 day) on which the rat uncovered the food well when it was $90 \%$ to $100 \%$ fully covered. All subjects met this criterion by the 3rd day of such training.

Discrimination training began on the day following completion of pretraining. Twenty trials were given each day, with position counterbalanced according to a Gellermann series. The rat was prevented from correction by lowering the door immediately after he had retracted his head from the chosen window. Both food wells were baited on each trial, with the incorrect stimulus locked. For the first task, the criterion was 18 correct choices on any one day. All subjects were then given 2 additional days of overtraining. If the subject failed to get 15 correct choices on either of the two overtraining days, training continued until he had again achieved the 18/20 criterion, and 2 days of overtraining were again administered. Only one subject failed to hold the criterion during overtraining.

Each subject was started on the second task on the day after it finished overtraining on the first task. Training on the shift task continued until the subjects had achieved 18 correct choices on any one day.

\section{Design}

One of the purposes of the present experiment requires a comparison of performances of counterbalancing subgroups. In order to obtain an adequate number of subjects in each subgroup, and to keep the number of subjects at a practical total, it was necessary to restrict the number of subgroups formed. This restriction was accomplished in part by assigning one set of stimuli to the first task and a second set to the second task, rather than to counterbalance stimuli across the two tasks. Although this procedure precludes comparison of first- and second-task performances, it does not affect the validity of ID and ED comparisons on Task 2. The number of subgroups was further restricted by making positive in Task 1 only one of the two possible cues on each relevant dimension. Since each cue on the second-task relevant dimension was positive for some subjects, the potential differential effects of generalized preferences can still be assessed. The design is summarized in Table 1 , where four pairs of ID and ED subgroups are described in terms of the 
Table 1

Description of First and Second Tasks for Pairs of ID and ED Subgroups

\begin{tabular}{|c|c|c|c|c|}
\hline \multirow[b]{2}{*}{ Subgroups } & \multicolumn{2}{|c|}{ Intradimensional Shift } & \multicolumn{2}{|c|}{ Extradimensional Shift } \\
\hline & Task 1 & Task 2 & Task 1 & Task 2 \\
\hline 1 & form-circle & form-square & stripes-vertical & form-square \\
\hline 2 & form-circle & form-X & stripes-vertical & form-X \\
\hline 3 & stripes-vertical & stripes-wide & form-circle & stripes-wide \\
\hline 4 & stripes-vertical & stripes-narrow & form-circle & stripes-narrow \\
\hline
\end{tabular}

nature of their first and second tasks. The first member of the hyphenated pair in Table 1 refers to the dimension relevant for a given task, and the second member refers to the positive cue for that task. All subjects thus received as their first discrimination either a form-relevant/circle-positive or a stripes-relevant/ vertical-positive task. The first-task negative cues were horizontal stripes or inverted triangle. The ID and ED pairs of subgroups received identical second tasks.

\section{RESULTS}

For each subject, the number of errors through criterion was determined separately for the two tasks. Following Shepp and Eimas (1964), these scores were transformed to logarithms, thereby reducing the degree of positive skew in the distributions. The antilogarithms of the various subgroup means are the geometric means of the original distributions.

\section{Original Learning}

The transformed errors in Task 1 were analyzed in a two-way factorial analysis of variance, with dimension relevant (stripes vs. forms) and type of shift (ID vs. ED) as the two factors. The geometric mean for the task with stripe orientation as relevant (81.5) was found to be significantly greater $[F(1,28)=41.7, p<.001]$ than that with form as relevant (23.1). The remaining two effects in this analysis were not significant $(p>.05)$. It may be noted that the geometric mean number of errors on Task 1 for all subjects in the present experiment (43.4) is somewhat less than half the number (90.4) reported by Shepp and Eimas.

\section{Shift Performance}

Table 2 presents the geometric-mean errors and sample standard deviations in Task 2 for the subgroups defined in Table 1. There it may be seen that three of the four ID subgroups averaged fewer errors to criterion than did their corresponding ED subgroups. The trans-

Table 2

Geometric-Mean Errors and Standard Deviations of Subgroups of ID and ED Shift Groups During Transfer

\begin{tabular}{cccccc}
\hline & \multicolumn{2}{c}{ Intradimensional } & & \multicolumn{2}{c}{ Extradimensional } \\
\cline { 2 - 3 } \cline { 5 - 6 } Subgroups & Mean & SD & & Mean & SD \\
\hline 1 & 5.9 & 10.5 & & 19.2 & 4.6 \\
2 & 80.1 & 47.3 & 73.6 & 14.3 \\
3 & 29.1 & 36.3 & 76.8 & 40.2 \\
4 & 44.7 & 24.9 & & 77.9 & 24.0 \\
Combined & 28.0 & 32.7 & & 53.9 & 24.6 \\
\hline
\end{tabular}

formed scores were subjected to a two-way analysis of variance in which type of shift and subgroups were the factors. Both type of shift $[F(1,24)=6.0, p<.02]$ and subgroups $[\mathrm{F}(3,24)=10.9, \mathrm{p}<.001]$ were significant. The critical interaction between these two factors was not significant $[F(3,24)=1.08, p>.25]$. Analysis of the raw scores provided the same pattern of results.

\section{DISCUSSION}

Despite several differences in apparatus, procedures, stimuli, and subject population, the present experiment confirms the results obtained by Shepp and Eimas (1964). Although the present data appear to be considerably more variable than those of Shepp and Eimas, there is a rather remarkable correspondence between the two sets of means for the ID and ED transfer conditions. Where Shepp and Eimas found geometric mean errors of 23.2 and 56.0 for the ID and ED shift performance, the present values were 28.0 and 53.9 .

Although the magnitude of the ID-ED difference varied somewhat among the counterbalancing subgroups, this variation was well within the range expected, given the basic variability in the data. There is no evidence, therefore, that the general ID superiority was produced by favorable generalization of the first-task instrumental response for some subgroups of ID subjects. The present investigators therefore conclude that they have replicated the basic finding of Shepp and Eimas and, given the assessment of differential transfer of the instrumental response, have rendered implausible the interpretation that ID superiority is produced solely by favorable generalization of the instrumental response.

\section{REFERENCES}

Kendler, H. H., Kendler, T. S., \& Ward, J. W. An ontogenetic analysis of optional intradimensional and extradimensional shifts. Journal of Experimental Psychology, 1972, 95, 102-109.

Mackintosh, N. J. The psychology of animal learning. New York: Academic Press, 1974.

Rollin, A. R., Shepp, B. E., \& Thaller, K. A modified Wisconsin General Test Apparatus for the rat. American Journal of Psychology, 1963, 76, 500-502.

ShePr, B. E., \& Eimas, P. D. Intradimensional and extradimensional shifts in the rat. Journal of Comparative and Physiological Psychology, 1964, 57, 357-361.

Shepp, B. E., \& TURRISI, F. D. Learning and transfer of mediating responses in discrimination learning. In N. R. Ellis (Ed.), International review of research in mental retardation (Vol. 2). New York: Academic Press, 1966. Pp. 86-120.

Turrisi, F. D., Shepp, B. E., \& Eimas, P. D. Intra- and extra-dimensional shifts with constant- and variableirrelevant dimensions in the rat. Psychonomic Science. $1969,14,19-20$. 